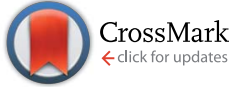

Cite this: RSC Adv., 2017, 7, 11665

Received 25th January 2017

Accepted 10th February 2017

DOI: 10.1039/c7ra01160a

rsc.li/rsc-advances

\section{Can sodium silicates affect collagen structure during tanning? Insights from small angle X-ray scattering (SAXS) studies $\uparrow$}

\author{
Yi Zhang, ${ }^{a}$ Bridget Ingham, ${ }^{b}$ Jérôme Leveneur, ${ }^{\text {ce }}$ Soshan Cheong, ${ }^{d}$ Yin Yao, ${ }^{d}$ \\ David J. Clarke, ${ }^{b}$ Geoff Holmes, ${ }^{a}$ John Kennedy ${ }^{c e}$ and Sujay Prabakar*a
}

\begin{abstract}
The effect of sodium silicates on collagen structure during leather processing was investigated. Small angle $X$-ray scattering (SAXS) and differential scanning calorimetry (DSC) reveal that the molecular structure and thermal stabilities of the sodium silicate treated leathers (So-Si and So-Si + BCS) were different to the conventionally processed chromium treated leathers (BCS). The collagen fibrils were observed to be coated by aggregates of silica, which did not affect the axial periodicity ( $D$-period) of the collagen molecules. However, an increase in collagen fibril diameter was observed during the main tanning step when sodium silicates were used. This could be due to the swelling of collagen fibers from the high alkaline conditions of sodium silicates. From DSC studies, it was also found that sodium silicate treated samples impart no effect on collagen stabilization in the absence of chromium(III). However, a pseudostabilization effect is observed in the So-Si + BCS samples, possibly due to the inability of the collagen molecules to undergo conformational changes due to the silica coating on the collagen fibrils.
\end{abstract}

The tanning of leather involves chemically intense processes leading to environmental pollution, resulting in a demand for cleaner but effective collagen stabilization mechanisms for the leather industry. ${ }^{\mathbf{1} 2}$ Basic chromium(III) sulphate is the most common mineral tanning agent and, is preferred industrially because of the high hydrothermal stability. It has excellent properties in addition to relative short times required to produce finished leathers. ${ }^{3}$ However, poor uptake of chromium salts leads to high chemical and biological oxygen demand, and toxicity concerns relating to hexavalent chromium(vi) exposure, have led researchers to seek more environmentally friendly alternatives. $^{\text {4-6 }}$

Mineral tanning agents such as chromium, zirconium, aluminium, titanium and iron can all stabilize collagen and impart varying degrees of hydrothermal stability. ${ }^{3,7}$ Synthetic tanning agents (syntans) and vegetable tanning agents from plant polyphenols along with aldehydic cross-linkers are also

${ }^{a}$ Leather and Shoe Research Association of New Zealand, P. O. Box 8094, Palmerston North 4472, New Zealand. E-mail: sujay.prabakar@lasra.co.nz

${ }^{b}$ Callaghan Innovation, P. O. Box 31310, Lower Hutt 5040, New Zealand

${ }^{c}$ National Isotope Centre, GNS Sciences, P. O. Box 31312, Lower Hutt 5040, New Zealand

${ }^{d}$ Electron Microscope Unit, Mark Wainwright Analytical Centre, University of New South Wales, Sydney, NSW 2052, Australia

${ }^{e}$ MacDiarmid Institute of Advanced Materials and Nanotechnology, Victoria University of Wellington, P. O. Box 600, Wellington 6140, New Zealand

$\dagger$ Electronic supplementary information (ESI) available: Experimental details, DSC, plots, additional SAXS patterns and characterization techniques. See DOI: 10.1039/c7ra01160a commonly used, but typically in conjunction with mineral tannages. $^{8}$ Combination tannages however, with or without mineral tanning agents, can overcome the issues that single tanning systems have with hydrothermal stability., ${ }^{\mathbf{9 1 0}}$ For example, Vitolo and co-workers observed an increase in chromium uptake when sodium silicate was used in combination with basic chromium sulphate. ${ }^{\mathbf{1 1}}$ Whilst pre-tans can improve the penetration and even distribution of the main tanning agents in the collagen matrix, a co-stabilizing agent can increase the efficiency of chrome uptake by reducing the amount of chrome required. ${ }^{8}$ With this idea of combining a weak and strong tanning agent, further studies have been carried out by investigating combinations of a number of tanning options and their effect on collagen stabilization. , $^{\mathbf{4 , 9 , 1 2}}$

Soluble silicates such as sodium silicate belong to a group of compounds that contain varying compositions of an alkali metal or quaternary ammonium salt and silica with water. ${ }^{13}$ For many years, the idea of stabilizing collagen with silica has been pursued with intent as it offers a cheap and environmentally friendly route to tanning. ${ }^{\mathbf{1 4 , 1 5}}$ Munz and co-workers demonstrated the ability of sodium silicate to be substituted for lime in the un-hairing process, in addition to improving the uptake of subsequent tanning agents. ${ }^{16,17}$ Rao and co-workers similarly showed that an enzymatic un-hairing process could be enhanced using sodium metasilicate. ${ }^{\mathbf{1 , 1}}$ Both Munz and Rao observed a mild stabilization effect but did not report any changes in the denaturation temperature. Coradin and coworkers extensively studied the interactions of various silica 
containing compounds with collagen for biomedical applications. ${ }^{19}$ However, very few studies have investigated the interactions of soluble silicates with collagen, with the aim of exploring alternative crosslinking chemistries for the leather processing industry. ${ }^{\mathbf{1 1 , 1 6 - 1 8}}$ In our study, we report for the first time, small angle X-ray scattering studies on sodium silicate treated leather and discuss a possible mechanism for its stabilization.

To study the effect of sodium silicates in leather processing we treated pickled NZ lambskins with a conventional chrome process using basic chromium sulphate (BCS), sodium silicate (So-Si) or sodium silicate/basic chromium sulphate (So-Si + BCS). Detailed processing steps and experimental methodologies are presented in the ESI. $\dagger$ Experiments were conducted on samples at each stage of the leather process, from pickling up to the finished leather. Pickling (stage 1) involves the acidification of collagen molecules in order to protonate the carboxyl groups, to prepare for chrome tanning and it also acts as a preservation step for longer term storage. ${ }^{8}$ In stage 2 , we then treated the pickled pelts with the pre-tanning agent Zoldine ZE (oxazolidine E), a five-membered heterocyclic compound derived from the reaction between an amino-hydroxy compound and aldehyde. ${ }^{20,21}$ Pre-tanning agents such as Zoldine ZE are commonly used to improve the penetration of main tanning agents through the collagen matrix in the skin, by decreasing the availability of amino acid side chain groups on the collagen molecule. When the samples were treated with the main tanning agents BCS, So-Si or So-Si + BCS (stage 3), different rates of fixation were observed based on the colour of the floats and the cross-sections of the skins. Whilst BCS treated samples revealed a uniform penetration of the chromium salts characterized by a blue centre, a cross-section of the So-Si samples exhibited a translucent centre akin to un-tanned collagen. The So-Si + BCS samples although initially consisting of a translucent centre, exhibited a blue cross-section after treating with BCS. Further, So-Si + BCS samples were found to have a well exhausted float, indicating that the So-Si samples enabled good exhaustion of subsequent tanning agents. Re-tanning (stage 4) was done by mimosa, a plant polyphenol derivative normally used to impart additional properties such as increased chemical and hydrothermal stabilities, organoleptic properties and better appearance. $^{8}$ The fatliquoring (stage 5) process prevents collagen fibres sticking together with each other as well as imparting softness by introducing oils and fats ${ }^{8}$ and was carried out using Chromapol SG and Polyol AK. At both these stages no remarkable changes in collagen structure or changes in denaturation temperature were observed. After this the samples were washed (stage 6) and held for further characterization prior to drying (stage 7).

Cross-sections of the samples treated with BCS, So-Si and SoSi + BCS were imaged using SEM and are presented in Fig. 1. The BCS samples showed a uniform and well dispersed collagen fibre structure. For both So-Si and So-Si + BCS samples the fibres were observed to be covered in a non-uniform coating of silica particles (Fig. 1B and C). This coating was made up of silica and at a higher magnification revealed that the silica had formed random aggregates of particles on the surface of the fibres in addition to coating them. The electrostatic interactions between positively charged amino groups of collagen with negatively charged silicate species can lead to rapid precipitation, forming particles of silica on the surface of the collagen fibrils.

Small-angle X-ray scattering (SAXS) experiments were performed on samples isolated at various stages of leather processing. These enabled us to study the long-range interactions
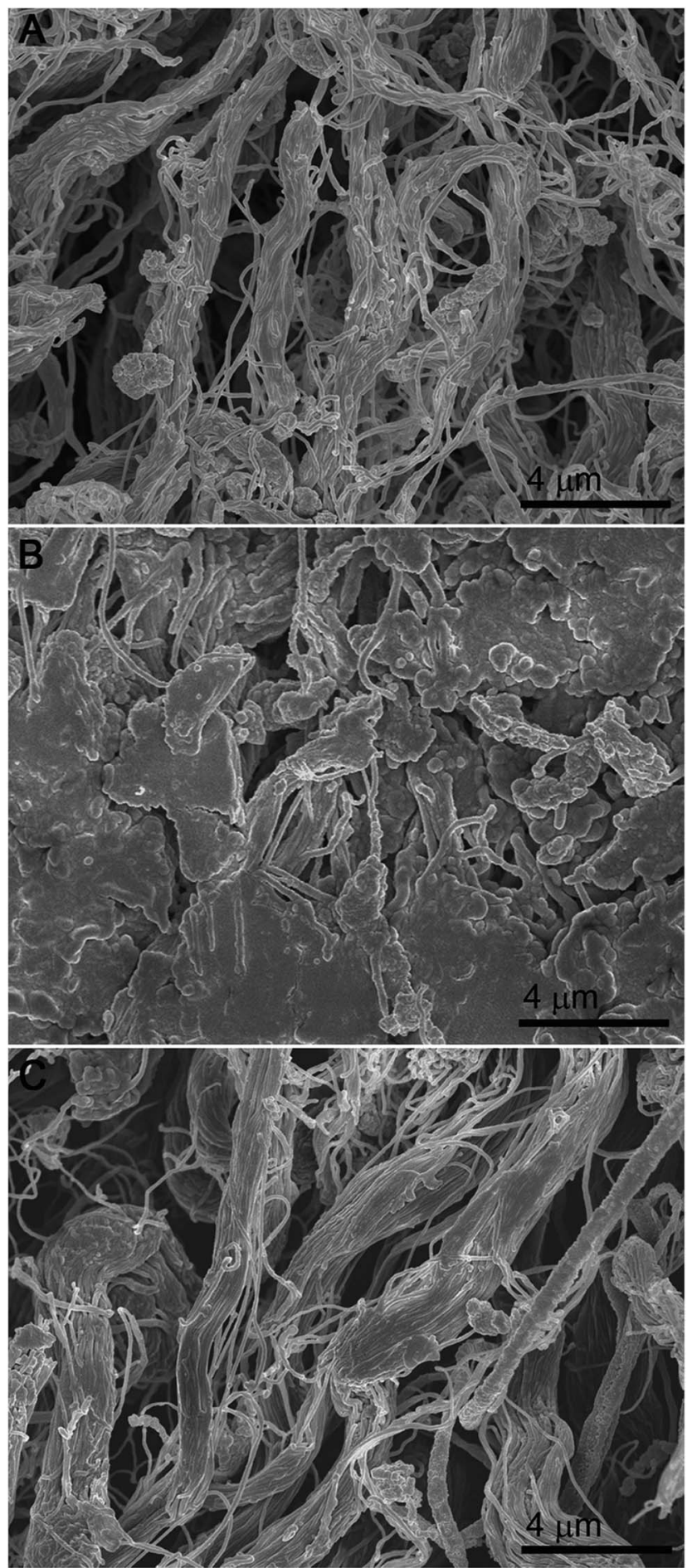

Fig. 1 SEM images of the cross-sections of (A) BCS, (B) So-Si and (C) So-Si + BCS finished leather samples. 

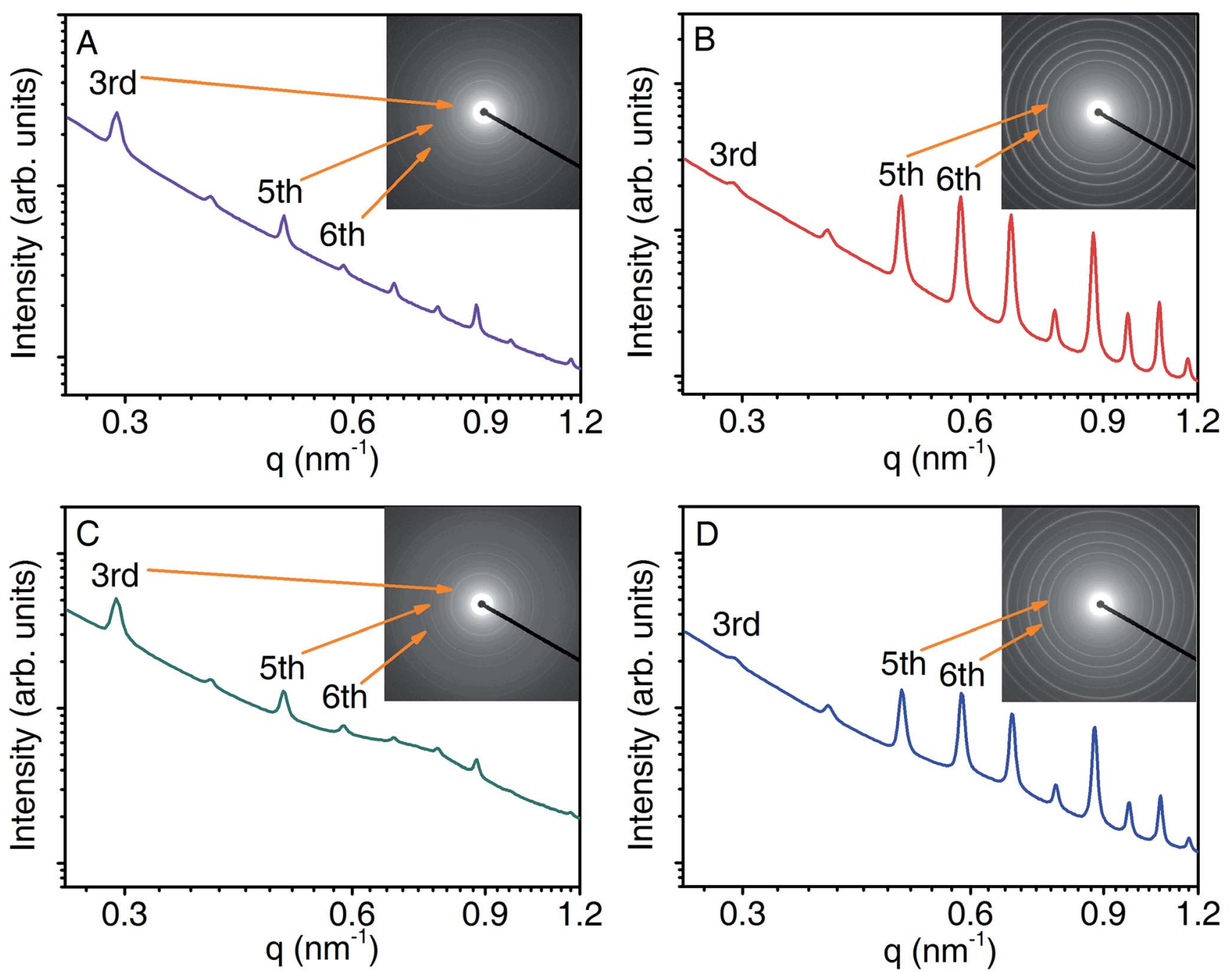

Fig. 2 SAXS diffraction patterns from (A) pre-tanned (stage 2), (B) BCS, (C) So-Si, (D) So-Si + BCS treated samples (stage 3). Selected peaks corresponding to $q=2 \pi i / D$ where $i$ is the peak order and $D$ the axial period, are labelled.

of collagen resulting from the axial order. To study the effect of the main tanning agents on the collagen structure we first compare the differences in scattering intensities between untanned (pickled and pre-tanned samples, stage 1 and 2), BCS, So-Si and So-Si + BCS samples (stage 3). The scattering profiles for both pickled and pre-tanned samples (see ESI-1†) were similar to each other and exhibited the typical isotropic collagen X-ray diffraction patterns of ovine skins, displaying a series of Bragg peaks arising from the $D$-period (Fig. 2A). The scattering profile of the BCS treated leather sample (Fig. 2B) revealed significant changes in the relative diffraction intensities of the Bragg peaks, indicating that the internal structure of the collagen molecules had changed. Maxwell and co-workers observed a similar effect in chromium tanned bovine hide and attributed the apparent increase in scattering intensity to the increased electron density contrast, due to the introduction of $\mathrm{Cr}(\mathrm{III})$ ions. $^{22}$ The So-Si treated samples (Fig. 2C) resembled un-tanned skins (ESI-1 $\dagger$ ) with an additional diffuse peak observed in the high $q$ region $\left(0.5-1 \mathrm{~nm}^{-1}\right)$. This peak is most likely due to the presence of silica particles adsorbed onto the surface of the collagen fibrils, ${ }^{23}$ produced by the hydrolysis of sodium silicate. So-Si + BCS samples exhibited both similar collagen diffraction to the BCS sample and a diffuse peak from the silica particles, indicating that sodium silicate interactions with the collagen did not interfere with the chrome tanning reaction.

The changes in $D$-period with leather processing are presented in Fig. 3A. When pickled skins are treated with the pretanning agent (Zoldine ZE, stage 2) a slight decrease in the $D$ period ( $65.5 \mathrm{~nm}$ to $64.5 \mathrm{~nm}$ ) is observed. The aldehyde groups of Zoldine ZE are known to react with the amine groups of lysine to form imines, that subsequently cross-link by means of covalent bonds with primary amine groups and other amino acid residues. $^{24}$ This can cause changes in the overall gap/overlap regions of the collagen molecule, in our case leading to a decrease in the $D$-period. However, during main tanning (stage 3) for all three treatments, no such changes in $D$-period were observed. In the case of the BCS treated samples, the kinetics of the chrome-collagen reaction is dependent on the availability of reactive sites on collagen. 

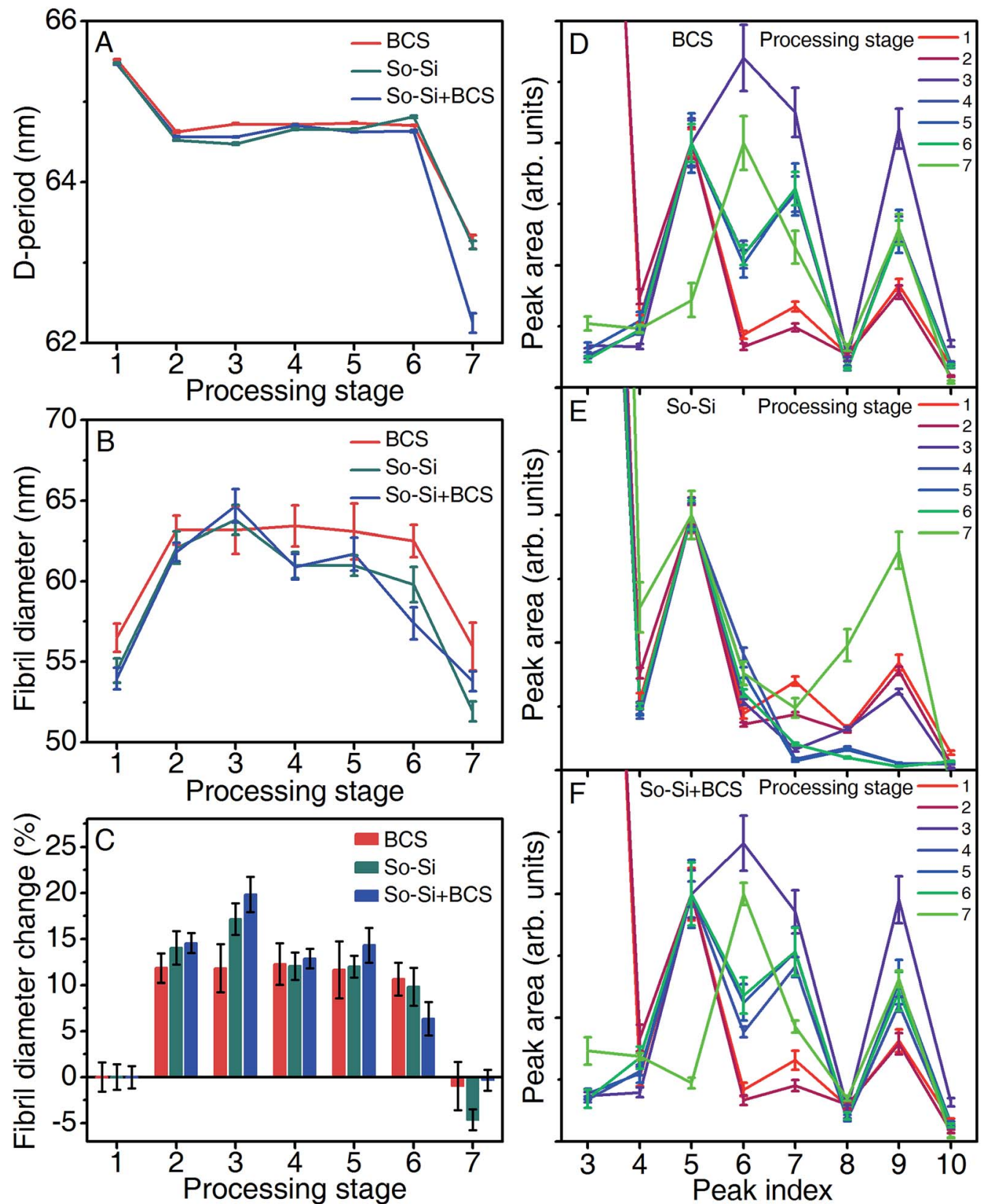

Fig. 3 Left: SAXS analysis of BCS, So-Si and So-Si + BCS skin samples after various leather processing stages (1) pickling, (2) pre-tanning, (3) main tanning, (4) re-tanning, (5) fat-liquoring, (6) wet leather (crust), (7) dry leather (crust) showing (A) change in D-period with processing, (B) variation in fibril diameter with processing and $(C)$ corresponding percentage change in fibril diameter. Right: scaled relative peak areas versus peak index for various leather processing stages as above, for (D) BCS, (E) So-Si and (F) So-Si + BCS treatments.

Also, pre-tanning with Zoldine ZE is understood to enhance the uptake of chromium salts by making carboxylic acid side chains of the glutamic acid and aspartic acid residues more accessible to chrome, thereby enhancing its even distribution and fixation in the collagen matrix. ${ }^{21}$ We speculate that the axial periodicities are unchanged because of the exhaustion of terminal amino acid side chain groups by the Zoldine ZE. Maxwell and co-workers observe an increase in $D$-period with the addition of chromium salts in the absence of Zoldine $\mathrm{ZE},{ }^{22,25}$ further reinforcing the idea that chromium interactions with collagen is specific to carboxylic acid groups. Further leather processing stages (4-6) did not cause any significant changes in the $D$-period as can be seen from Fig. 3A. However, a sharp decrease in the $D$-period was observed in the finished leather samples on drying (stage 7). Drying of finished leathers was done at $45{ }^{\circ} \mathrm{C}$ to remove unbound water (or free water) from the collagen matrix in leather, resulting in a finished dry leather containing $8-12 \%$ moisture (determined using a moisture meter (Aqua-Boy)). Unlike bound intermolecular water (or structural water), which is relatively hard to remove from collagen, unbound water can be removed by air-drying, which is known to cause the collagen structure to collapse. ${ }^{26}$ Free water 
affects the intermolecular lateral packing in collagen molecules and when removed by drying is understood to cause a decrease in the axial periodicity of the molecule by a collapse in the gap/ overlap region in the collagen molecule and the partial shearing of unit cell contents within the gap region upon loss of water. ${ }^{27}$ Fig. 3B and $\mathrm{C}$ show changes in fibril diameter and their corresponding percentage differences with leather processing. Prior to treating with the main tanning agents, at both pickling and pre-tanning stages (1-2 respectively) the samples are observed to have similar fibril diameters. The BCS treated samples (63.2) were tanned directly at a $\mathrm{pH}$ of $\sim 4.5$, showing no changes in fibril diameter. During the main tanning, the sodium silicate treated samples, So-Si and BCS + So-Si show an increase in fibril diameter $(62.1 \mathrm{~nm}$ to $63.1 \mathrm{~nm}$, and $61.9 \mathrm{~nm}$ to $64.6 \mathrm{~nm}$ respectively). This could be due to the high $\mathrm{pH}(>11)$ conditions that develop in the sodium silicate solutions, leading to a weakening of the intermolecular interactions between the collagen molecules and consequently an ingress of unbound water, resulting in swelling. Maxwell and co-workers observed similar changes in fibril diameters at high alkalinity but during the liming stage. ${ }^{25}$ No appreciable differences in fibril diameter were observed after the main tanning step between the three samples although depicted in Fig. 3B as having a range of diameters $(57-63 \mathrm{~nm})$, the percentage differences (Fig. 3C) indicate that the differences among treatments are not significant. Upon air-drying (stage 7), a concomitant decrease in fibril diameter is observed for all samples, which is to be expected because of the collapse of the intermolecular lateral packing of the collagen structure. Fig. 3D-F shows the relative diffraction peak intensities for each reflection for each sample after the different tanning stages. The different patterns arise from differences in electron density contrast along the collagen repeat unit. ${ }^{28,29}$ These can be affected by the presence or absence of water, salts, etc. ${ }^{30}$ There is relatively little change between the pickled and pre-tanned samples (stage 1 and 2). After the main tanning (stage 3), there are significant changes for the BCS and So-Si + BCS treated samples: the 6th, 7th and 9th order peak intensities increase. However, the change in the So-Si sample is minor (the 7th order peak decreases in intensity, while the other peaks are relatively unchanged). After re-tanning (stage 4) there is another significant change in the BCS and So-Si + BCS samples: the 6th order peak reduces in relative intensity, while for the So-Si sample there are subtle changes in the intensities of the 6th and 9th peaks. Between stages 4-6 there are negligible changes for all samples. Upon drying (stage 7) the intensity of the 5th peak decreases and the 6th peak increases for the BCS and So-Si + BCS samples, while for the So-Si sample the relative intensities of the 5th and 6th peaks remains constant while the 8th and 9th relative peak intensities increase. It is not trivial to assign these changes to specific structural rearrangements of the collagen molecule, Cr salts, bound and free water, etc. From this examination, we conclude that the silicate treatment by itself does not alter the collagen structure at all, and also does not interfere with the structural changes induced through $\mathrm{Cr}(\mathrm{III})$ treatment.

Tapping mode atomic force microscopy (AFM) was used to further characterize the collagen fibrils. Fig. 4 displays typical deflection amplitude images of the three dry leather samples. These images show banding along the collagen fibrils arising from the $D$-period. In some images and at random locations of the So-Si and the So-Si + BCS samples, random indentations (white box) on the surface of the fibrils were observed, which we attribute to the morphological changes brought upon the collagen fibrils by the high alkaline conditions $(\mathrm{pH}>11)$ of the sodium silicates. Such features are observed clearly in Fig. 4B and to a lesser extent in Fig. 4C. The $D$-period values obtained from 2D FFT analysis of the AFM images are larger than those
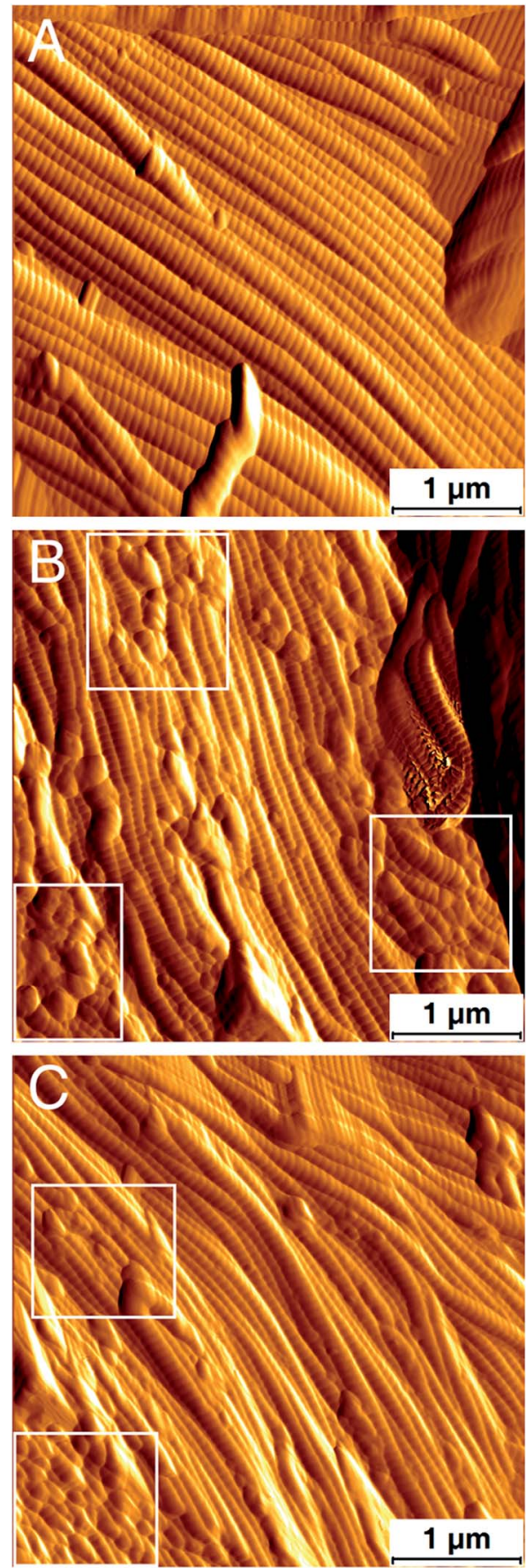

Fig. 4 Atomic force microscopy amplitude of deflection images of (A) BCS, (B) So-Si samples and (C) So-Si + BCS. 
Table 1 DSC analysis of the BCS, So-Si and So-Si + BCS samples at different processing stages

\begin{tabular}{llll}
\hline & \multicolumn{3}{l}{ Denaturation temperature $T\left({ }^{\circ} \mathrm{C}\right)$} \\
\cline { 2 - 4 } Processing stage & BCS & So-Si & So-Si + BCS \\
\hline Pickling (1) & 56 & 55 & 56 \\
Pre-tanning (2) & 75 & 73 & 73 \\
Main tanning (3) & 110 & 74 & 111 \\
Re-tanning (4) & 113 & 79 & 111 \\
Fatliquoring (5) & 114 & 79 & 110 \\
Wet leather (6) & 114 & 83 & 116 \\
Dry leather (7) & 315 & $215 / 278$ & 335
\end{tabular}

from SAXS, which could be explained on the basis that AFM is a surface technique while SAXS is a volume technique, in addition to the effect of hydration during AFM sample preparation. $^{27,31}$ The role of sodium silicates in stabilizing the collagen structure in leather was further investigated by studying the hydrothermal stability of the samples using differential scanning calorimetry (DSC) (Table 1). After pretanning with Zoldine ZE an increase in hydrothermal stability of around $18{ }^{\circ} \mathrm{C}$ was observed for all samples, which is consistent with previous reports and can be attributed to the Zoldine ZE induced covalent cross-links between lysine and other amino acid groups on collagen. ${ }^{24}$ After tanning with BCS, an increase in denaturation temperature to $110{ }^{\circ} \mathrm{C}$ was observed. Chrome tanning is known to influence the association of bound water with collagen, resulting in an increase in the denaturation temperature. ${ }^{32}$ However, for the So-Si sample no significant change in denaturation temperature was observed. The So-Si + BCS sample had a denaturation temperature of $111{ }^{\circ} \mathrm{C}$ indicating again that the sodium silicates did not interfere with the chrome tanning mechanism. The denaturation temperature of dry finished leather samples was also studied to give us a better understanding of thermal stability (Table 1). Dry leather samples will have reduced configurational enthalpies of the thermally labile domains that determine the rates of denaturation in collagen, and are known to exhibit an increase in the denaturation temperature. ${ }^{26}$ The So-Si samples had a low denaturation temperature of $215{ }^{\circ} \mathrm{C}$, and an additional endothermic peak at $278{ }^{\circ} \mathrm{C}$ that could be assigned to the combination of the hydrogen-bonded water on silica particles and the condensation of germinal and vicinal hydroxyls of silanol groups from the silica particle coating. ${ }^{33}$ For the So-Si + BCS samples a denaturation temperature of $335^{\circ} \mathrm{C}$ was observed, compared to $315^{\circ} \mathrm{C}$ for the BCS samples. The small increase in denaturation temperature could be attributed to the ability of sodium silicates to form silica particles, confining the collagen molecules and inhibiting them from undergoing conformational changes. ${ }^{34}$ We also speculate that the silica coating on the collagen fibres limits the possibility of protein unfolding, inadvertently affecting denaturation temperature. ${ }^{34}$

Chemical characterization of the dry leather samples was performed by FTIR analysis. Typical results are presented in Fig. 5 for the BCS, So-Si and So-Si + BCS samples. The presence of silica in the leather samples could be determined by the

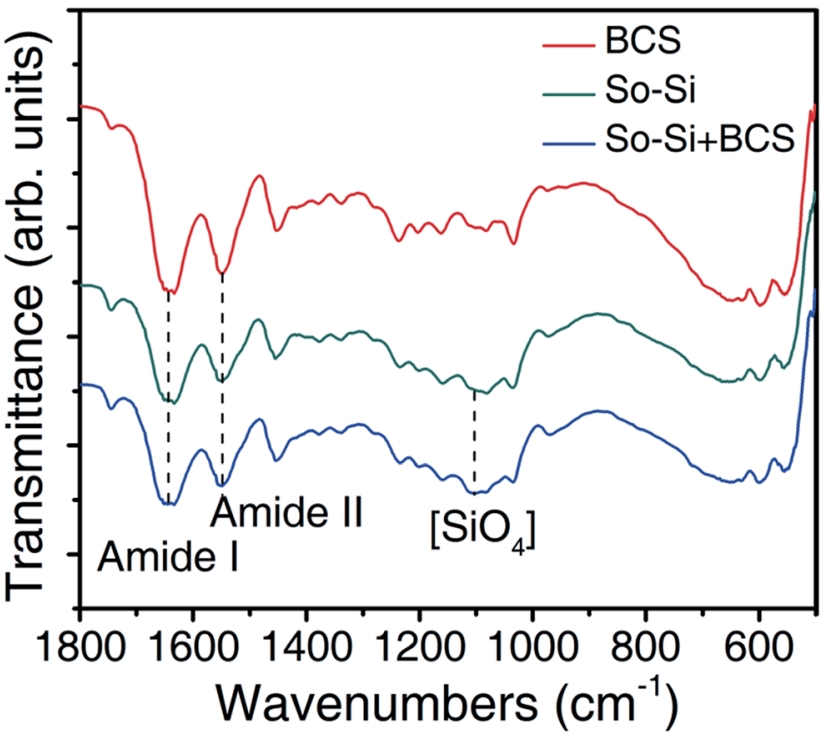

Fig. 5 FTIR spectra of BCS, So-Si and So-Si + BCS samples after drying (stage 7).

stretching vibration mode of the $\left[\mathrm{SiO}_{4}\right]$ tetrahedron $\left(1100 \mathrm{~cm}^{-1}\right)$ observed in both So-Si and So-Si + BCS samples, ${ }^{35}$ while the characteristic peaks of the collagen backbone could be identified by the amide bands (amide II, $1640 \mathrm{~cm}^{-1}$; amide I, $1550 \mathrm{~cm}^{-1}$ ). ${ }^{36}$

\section{Conclusions}

In summary, the effect of sodium silicates on the collagen structure during leather processing has been investigated by SEM, SAXS and DSC. We speculate that the electrostatic interactions between the positively charged amino groups of collagen with negatively charged silicate species lead to precipitation, forming coatings and aggregates of silica particles on the surface of the collagen fibrils. The introduction of silica into the leather matrix did not affect the axial periodicities of the collagen molecules, however an increase in collagen fibril diameter was observed during the main tanning step. This could be due to the swelling of collagen fibres from the high alkaline conditions of sodium silicates. From DSC studies, it was found that sodium silicate treated samples (So-Si) impart no effect on collagen stabilization in the absence of BCS. However, a pseudo-stabilization effect is observed in the So-Si + BCS samples possibly due to the inability of the collagen molecules to undergo conformational changes due to the silica coating on the collagen fibrils.

\section{Acknowledgements}

S. P., Y. Z. \& G. H. would like to thank the Ministry of Business, Innovation and Employment (MBIE) for funding through grant LSRX-1301. S. P. and Y. Z. thank Dr Anna Henning for assistance with electron microscopy analysis. Portions of this work were conducted on the SAXS beamline at the Australian Synchrotron, Victoria, Australia. Part of this research used the facilities at the Electron Microscope Unit at UNSW. 


\section{Notes and references}

1 K. Sreeram and T. Ramasami, Resour., Conserv. Recycl., 2003, 38, 185-212.

2 Y.-N. Wang, Y. Zeng, J. Zhou, W. Zhang, X. Liao and B. Shi, J. Cleaner Prod., 2016, 112, 2-8.

3 A. D. Covington, Chem. Soc. Rev., 1997, 26, 111-126.

4 N. N. Fathima, B. Madhan, J. R. Rao and B. Nair, J. Am. Leather Chem. Assoc., 2003, 98, 139-146.

5 L. Yan, L. Zhaoyang, F. Haojun, L. Yuansen, L. Hui, P. Biyu and S. Bi, J. Soc. Leather Technol. Chem., 2008, 92, 252-257.

6 N. Fathima, J. Rao and B. Nair, J. Soc. Leather Technol. Chem., 2003, 87, 227-232.

7 A. Bacardit, S. van der Burgh, J. Armengol and L. Ollé, J. Cleaner Prod., 2014, 65, 568-573.

8 A. D. Covington and T. Covington, Tanning chemistry: the science of leather, Royal Society of Chemistry, 2009.

9 S. Cao, B. Liu, B. Cheng, F. Lu, Y. Wang and Y. Li, J. Hazard. Mater., 2017, 321, 203-209.

10 J. Shi, R. Puig, J. Sang and W. Lin, J. Cleaner Prod., 2016, 139, 1512-1519.

11 A. D'Aquino, G. D'Elia, M. Seggiani, S. Vitolo, B. Naviglio and M. Tomaselli, J. Am. Leather Chem. Assoc., 2004, 99, 11.

12 G. Krishnamoorthy, S. Sadulla, P. K. Sehgal and A. B. Mandal, J. Cleaner Prod., 2013, 42, 277-286.

13 H. H. Weldes and K. R. Lange, Ind. Eng. Chem., 1969, 61, 2944.

14 A. Rose, Can. J. Res., Sect. A, 1939, 17, 385-389.

15 H. P. van Dokkum, J. H. J. Hulskotte, K. J. M. Kramer and J. Wilmot, Environ. Sci. Technol., 2004, 38, 515-521.

16 K. Munz and R. Sonnleitner, J. Am. Leather Chem. Assoc., 2005, 100, 66-75.

17 K. H. Munz, J. Am. Leather Chem. Assoc., 2003, 98, 159-167.

18 S. Saravanabhavan, P. Thanikaivelan, J. R. Rao, B. U. Nair and T. Ramasami, Environ. Sci. Technol., 2008, 42, 17311739.

19 T. Coradin, O. Durupthy and J. Livage, Langmuir, 2002, 18, 2331-2336.
20 S. D. Choudhury, S. DasGupta and G. E. Norris, Int. J. Biol. Macromol., 2007, 40, 351-361.

21 S. Sundarapandiyan, P. E. Brutto, G. Siddhartha, R. Ramesh, B. Ramanaiah, P. Saravanan and A. Mandal, J. Hazard. Mater., 2011, 190, 802-809.

22 C. A. Maxwell, K. Smiechowski, J. Zarlok, A. Sionkowska and T. J. Wess, J. Am. Leather Chem. Assoc., 2006, 101, 9-17.

23 T. Gerber, B. Himmel and C. Hübert, J. Non-Cryst. Solids, 1994, 175, 160-168.

24 S. Deb Choudhury, R. G. Haverkamp, S. DasGupta and G. E. Norris, J. Agric. Food Chem., 2007, 55, 6813-6822.

25 C. A. Maxwell, T. J. Wess and C. J. Kennedy, Biomacromolecules, 2006, 7, 2321-2326.

26 C. A. Miles and M. Ghelashvili, Biophys. J., 1999, 76, 32433252.

27 T. J. Wess and J. P. Orgel, Thermochim. Acta, 2000, 365, 119128.

28 W. Claffey, Biophys. J., 1977, 19, 63.

29 D. J. Hulmes, A. Miller, S. W. White and B. B. Doyle, J. Mol. Biol., 1977, 110, 643-666.

30 O. Antipova and J. P. Orgel, J. Biol. Chem., 2010, 285, 70877096.

31 A. D. Kemp, C. C. Harding, W. A. Cabral, J. C. Marini and J. M. Wallace, J. Struct. Biol., 2012, 180, 428-438.

32 N. N. Fathima, M. Baias, B. Blumich and T. Ramasami, Int. J. Biol. Macromol., 2010, 47, 590-596.

33 J. Fripiat and J. Uytterhoeven, J. Phys. Chem., 1962, 66, 800805.

34 M. F. Desimone, C. Hélary, S. Quignard, I. B. Rietveld, I. Bataille, G. J. Copello, G. Mosser, M.-M. Giraud-Guille, J. Livage and A. Meddahi-Pellé, ACS Appl. Mater. Interfaces, 2011, 3, 3831-3838.

35 M. Wilson, P. A. Madden, M. Hemmati and C. A. Angell, Phys. Rev. Lett., 1996, 77, 4023.

36 B. B. Doyle, E. Bendit and E. R. Blout, Biopolymers, 1975, 14, 937-957. 